\title{
Low-Energy Electron Diffraction during Pulsed Laser Annealing: A Time- Resolved Surface Structural Study
}

\section{Citation}

Becker, R. S., G. S. Higashi, and J. A. Golovchenko. 1984. Low-Energy Electron Diffraction During Pulsed Laser Annealing: A Time- Resolved Surface Structural Study. Physical Review Letters 52, no. 4: 307-310. doi:10.1103/physrevlett.52.307.

\section{Published Version}

doi:10.1103/PhysRevLett.52.307

\section{Permanent link}

http://nrs.harvard.edu/urn-3:HUL.InstRepos:29407048

\section{Terms of Use}

This article was downloaded from Harvard University's DASH repository, and is made available under the terms and conditions applicable to Other Posted Material, as set forth at http:// nrs.harvard.edu/urn-3:HUL.InstRepos:dash.current.terms-of-use\#LAA

\section{Share Your Story}

The Harvard community has made this article openly available.

Please share how this access benefits you. Submit a story.

\section{Accessibility}




\title{
Low-Energy Electron Diffraction during Pulsed Laser Annealing: A Time- Resolved Surface Structural Study
}

\author{
R. S. Becker, G. S. Higashi, and J. A. Golovchenko \\ Bell Laboratories, Murray Hill, New Jersey 07974
}

(Received 4 October 1983)

\begin{abstract}
Nanosecond structural changes in a crystal lattice during pulsed laser annealing have been measured by use of time-resolved low-energy electron diffraction. Low-energy electron diffraction is both structure and surface $(\sim 10 \AA)$ sensitive and can yield lattice temperatures from Debye-Waller-like extinction coefficients. Combining these with nanosecond time resolution provides a surface probe for short-time dynamical processes. The results demonstrate rapid formation of a liquid layer and subsequent surface recrystallization and cooling on (111) Ge.
\end{abstract}

PACS numbers: 79.20.Ds, 61.14.Fe, 63.90. $+\mathrm{t}$

In recent years a great deal of effort has been expended in the study of fast-pulse laser annealing of semiconductors. Both the properties of the final material as well as the fundamental processes responsible for the changes that occur are of continuing interest. The study of the fundamental processes has presented experimentalists with the challenging problem of characterizing states of condensed matter that exist for only the brief intervals $\left(10^{-14}\right.$ to $\left.10^{-6} \mathrm{sec}\right)$ during which the laser beam is interacting with the semiconductor or the system is relaxing towards equilibrium. It may well be expected that the development and refinement of time-resolved observational methods in this regime will insure continued advancement of our understanding of condensed materials in highly excited nonequilibrium situations.

Our interest in this problem has lately been directed towards observing the state of the immediate atomic surface through which the light enters the material. None of the time-resolved methods already brought to bear on the study of laser annealing (e.g., optical reflectivity and transmission,,$^{1-4} \mathrm{x}$-ray diffraction, ${ }^{5,6}$ secondharmonic generation, ${ }^{7}$ Raman scattering, ${ }^{8-10}$ time-of-flight mass spectrometry ${ }^{11}$ ) yields atomic structural information on the first few atomic layers. It is of course the transformations of this region which precede and may even stimulate the course of the laser-target interaction. Furthermore, a comprehensive understanding of the state of the surface under these conditions may lead to advances in stimulating chemical reactions at this interface.

It would appear that a natural probe of this region would be time-resolved low-energy electron diffraction (LEED). There have in fact been recent reports of high-energy electron-diffraction measurements with picosecond resolution in an electron microscope ${ }^{12}$ and a modified streak camera. ${ }^{13}$ Such high energies (10-100 keV), however, are not particularly convenient for probing only the surface region of a crystal. Hence in the following we describe preliminary results of a new apparatus designed to observe low-energy electron scattering from crystal surfaces during pulsed laser irradiation. We observe the (00) diffraction spot from a (111) germanium crystal and show how it is extinguished under conditions associated with laser annealing. We further discuss how the state of the surface can be temporally resolved from a study of the scattering intensity.

The experimental setup is illustratd in Fig. 1. It consists of three major components: an ultrahigh vacuum (UHV) system for conventional sample preparation and characterization, a pulsed laser for exciting the sample, and a pulsed LEED system for time-resolved measurements. A (111) germanium sample was Syton polished to a mirror finish and then placed in the UHV chamber which was capable of a base pressure of $2 \times 10^{-10}$ Torr after bakeout. Once in the vacuum chamber the surface was sputter cleaned by use of 0.5 to

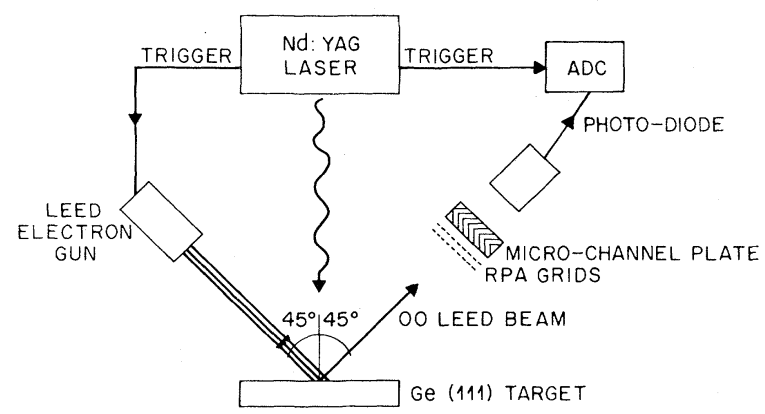

FIG. 1. Functional diagram of the apparatus indicating the relationship of the electron gun, Ge sample, microchannel-plate detector, laser, and counting electronics. 
$1.0 \mathrm{keV}$ argon ions and was subsequently thermally annealed at temperatures between 650 and $920^{\circ} \mathrm{C}$ with a radiative heater mounted behind the sample. After several of these annealing cycles the sample was observed, with conventional LEED, to exhibit the $2 \times 8$ diffraction pattern characteristic of a clean reconstructed Ge(111) surface. ${ }^{14}$ Upon laser annealing the surface reconstructs to $1 \times 1$, and hence this is the surface studied in this work.

The pulsed laser system consisted of a QuantaRay Q-switched Nd-doped yttrium-aluminumgarnet (YAlG) laser $(1.06 \mu \mathrm{m})$ which was operated with a pulse width of $9 \mathrm{nsec}$, at a repetition rate of $10 \mathrm{~Hz}$, in Quanta-Ray's standard unstableresonantor "doughnut" mode. In order to obtain a smooth Gaussian-like profile at the sample, the beam was propagated, with the aid of glass turning prisms and lenses, into its far field. At a beam diameter of $4 \mathrm{~mm}$, fluences on the sample as high as $500 \mathrm{~mJ} / \mathrm{cm}^{2}$ could be obtained. In order to assure that the laser pulse energy used in the time-resolved LEED experiment corresponded to that associated with the "laser annealing" regime, time-resolved optical reflectivity measurements were performed on a control sample outside the vacuum chamber. Figure 2 (a) shows the reflectivity of $P$-polarized light from a $\mathrm{He}-\mathrm{Ne} \mathrm{cw}$ laser $(632.8 \mathrm{~nm})$ incident at $30^{\circ}$ off the germanium surface normal. This probe beam was $0.2-0.3 \mathrm{~mm}$ in diameter and was centered directly on the region illuminated by the YAlG

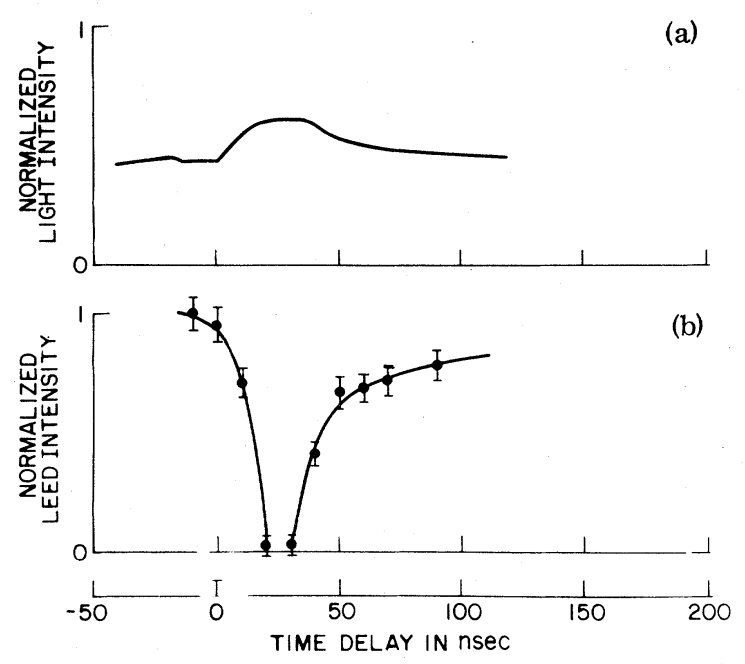

FIG. 2. (a) Reflectivity at $632.8 \mathrm{~nm}$ and (b) (00) beam LEED intensity at $75 \mathrm{eV}$ vs time on $\mathrm{Ge}(111)$, taken at a pump fluence of $390 \mathrm{~mJ} / \mathrm{cm}^{2}$. annealing pulse. The annealing pulse fluence was $390 \mathrm{~mJ} / \mathrm{cm}^{2} .{ }^{15}$ The reflectivity is seen to rise from 0.4 to 0.6 upon arrival of the annealing pulse and endures for 20-30 ns. The slow rise and fall times may be attributed to the bandwidth of our electronic display apparatus. This reflectivity result is quite characteristic of the laser annealing regime and hence the time-resolved LEED results shown below have been taken at the same YAlG laser power.

The pulsed LEED apparatus consisted of a modified commerical LEED electron gun and a microchannel-plate electron-multiplier detector system capable of single electron detection with position resolution of $<0.1 \mathrm{~mm}$. The electron gun was mounted in the UHV chamber so that the electron beam was incident on the sample at an angle of $45^{\circ}$. At the working distance used a spot size on the sample of $10 \times 1.5 \mathrm{~mm}$ was obtained, and this was carefully positioned with the aid of electrostatic deflection plates to be centered on the 4$\mathrm{mm}$ spot illuminated by the YAlG laser. A $60-\mathrm{V}$ bias below cathode potential on the Wehnelt cylinder of the extration lens holds the electron gun normally off. Application of a $+60-\mathrm{V}$ pulse capacitively coupled to this electrode turns the beam on for the duration of the pulse, nominally $10 \mathrm{~ns}$. Synchronization between YAlG laser and electron beam pulses at the sample surface was achieved by using the variable $Q$-switch sync on the laser to trigger the electron gun pulser. Since the lowenergy electrons take 25 to $35 \mathrm{nsec}$ to travel from cathode to sample, the electron gun was in fact triggered before the laser actually fired. Changing the delay between electron and laser pulses allowed temporal sampling of the LEED intensity in a window extending from a few hundred nanoseconds before to $1000 \mathrm{nsec}$ after the light pulse.

For electron pulse durations of $10 \mathrm{nsec}$ at 75 to $150 \mathrm{eV}$ space-charge effects at the cathode limited the pulse charge in our experiments to $\sim 10^{4}$ electrons. Typical LEED reflection coefficients are $\sim 10^{-4}$ for a major reflection spot, yielding about one reflected electron per laser shot. A two-stage microchannel plate operated at saturation gain and coupled to a phosphor screen was used to amplify the reflected LEED electrons and convert each to a light pulse which was imaged on a silicon photodiode. Only a single LEED spot could be imaged at a time in the present setup and hence we shall only report on one here. The detector is also equipped with retarding grids to reject stray ions and secon- 
dary electrons.

Figure 2(b) shows the time-resolved intensity of the 75-eV, (00) LEED spot from the germanium sample irradiated by $390-\mathrm{mJ} / \mathrm{cm}^{2}$ YAlG laser pulses at $10 \mathrm{~Hz}$. Zero intensity corresponds to our measured background level. The LEED spot is seen to extinguish for a period of 10 to $20 \mathrm{nsec}$ reaching a maximum reduction of greater than 20:1 during the high-reflectivity phase in Fig. 2 (a). Reappearance of the diffracted beam is equally abrupt, with recovery to $40 \%$ of the prepulse level in $10 \mathrm{nsec}$ and $65 \%$ in the next $10 \mathrm{nsec}$, and is followed by continued increases at a much slower rate.

Attenuation of diffracted beam intensity as the lattice temperature increases is well known in $x$-ray diffraction and is analyzed through the Debye-Waller coefficient which in turn is directly related to the excited vibrational modes of a bulk crystal. ${ }^{16}$ For LEED beams the extinction is vastly complicated by multiple scattering, inelastic effects, and unknown surface vibration modes, rendering a theoretical calculation of the temperature dependence of the effect nearly impossible. This problem is circumvented by performing an "in situ" experimental static calibration of LEED intensity versus temperature. The electron reflectivities in Fig. 2(b) may then be associated with temperatures determined from such a calibration.

Figure 3 shows the calibration curves resulting from such an exercise. Shown are the $(00)$ beam reflection intensities for electron energies of 75 and $140 \mathrm{eV}$ as a function of sample temperature. Here the sample was raised to each steady-state temperature by radiative heating from a filamenttype heater. The temperature is determined by thermocouple and optical pyrometer readings, and the LEED intensity was measured with use of the same apparatus used for the pulsed laser work. The 140-eV data are Debye-Waller like (i.e., they decrease exponentially with increasing temperature) except for a small knee at $\sim 285^{\circ} \mathrm{C}$, which may be connected to some phase transition in the $2 \times 8$ structure. The attenuation at $900{ }^{\circ} \mathrm{C}$ is about 100:1 relative to room temperature. ${ }^{17}$ The $75-\mathrm{eV}$ data are markedly different showing only slight attenuation from room temperature to $600^{\circ} \mathrm{C}$, with the intensity dropping much more rapidly between 600 and $920^{\circ} \mathrm{C}$. At $920^{\circ} \mathrm{C}$ the attenuation is $16: 1$ with $200^{\circ} \mathrm{C}$ as our baseline. A study of the magnitude of the error bars in our time-resolved LEED measurements shows that best accuracy at high temperatures is obtained

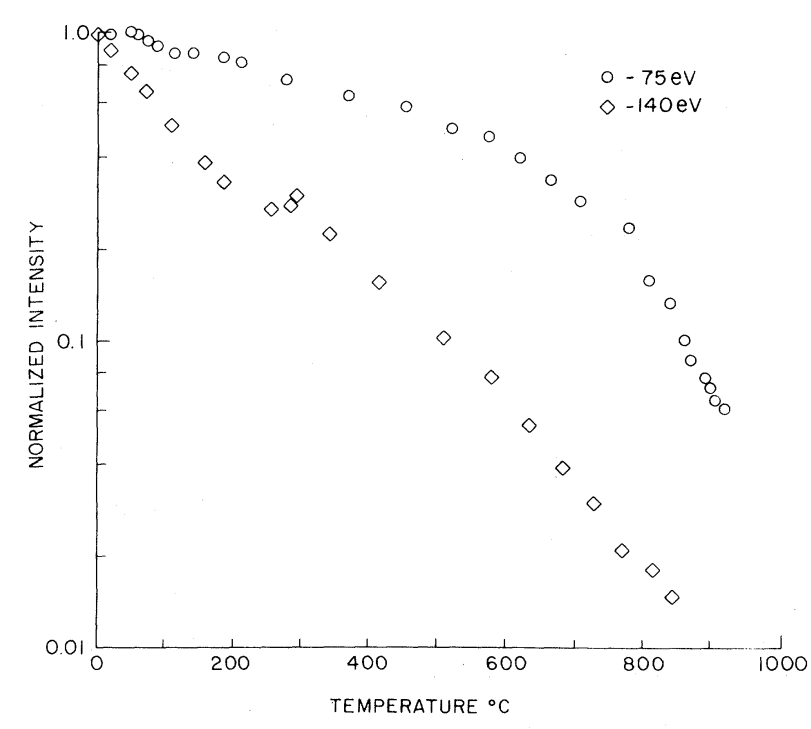

FIG. 3. (00) LEED intensity of Ge(111) as a function of temperature for electron energies of $75 \mathrm{eV}$ (upper curve) and $140 \mathrm{eV}$ (lower curve). All intensities are normalized to the room-temperature value.

with 75-eV electrons. Low temperatures, in which we are not currently interested, would best be studied with $140-\mathrm{eV}$ electrons.

Comparing the $75-\mathrm{eV}$ static temperature measurements with the time-resolved results we immediately see that the sample surface exhibits disorder exceeding that of germanium at its melting point $\left(937^{\circ} \mathrm{C}\right)$, since the observed extinction exceeds 20:1. After maximum extinction the surface reorders rapidly having a reflectivity similar to that of a $700^{\circ} \mathrm{C}$ surface after $10 \mathrm{nsec}$ and recovers to its steady-state value $\left(200^{\circ} \mathrm{C}\right)$ after a few hundred nanoseconds. Since LEED, at these energies, is primarily surface diffraction, the (00) beam exists as long as the surface is crystalline. Thus, the extinction observed clearly indicates that the Ge surface is noncrystalline during the laser annealing process. It may be possible to account for this intensity change by means other than melting, but it is difficult to construct an alternate model consistent with LEED and other time-resolved data. ${ }^{1-11}$

We believe that the above experimental results represent one of the most unambiguous means of observing the condition of the top atomic layers of a crystal during laser irradiation. While these preliminary studies were concerned with only a single reflection, because of detector size limitations, it is clear to us that improvements in the apparatus can easily yield entire $k$-space 
LEED patterns, with the attendant detailed information on surface crystal structure. There is a plethora of surface phase transitions, thermally stimulated chemical reactions, and desorption and diffusion processes that may now be probed during laser excitation by the electron-diffraction technique. We further believe that through suitable modification of the electron gun design the time scale of the experiments may be reduced into the picosecond regime where transient phases that are undetectable on the nanosecond time scale would be observable. It may be hoped that for excitations on shorter time scales, effects connected with high concentrations of hot carriers in a cold lattice will manifest themselves in new crystal lattice parameters and phases. It is only through the development of fast surface diffraction techniques that such new transient phases may be unambiguously identified.

We would like to acknowledge helpful discussions with D. H. Auston, B. Kincaid, E. McRae, and R. Malik of Bell Laboratories and Barney Webb of the University of Wisconsin. Rick Levesque made a valuable contribution to this work during its initial construction phase.

${ }^{1}$ D. H. Auston, J. A. Golovchenko, A. L. Simons, R. F. Slusher, P. R. Smith, C. M. Surko, and T. N. C. Venkatesan, in Laser-Solid Interactions and Laser Processing -1978 , edited by S. D. Ferris, H. J. Leamy, and J. M. Poate, AIP Conference Proceedings No. 50 (American Institute of Physics, New York, 1979).

${ }^{2}$ D. H. Lowndes, R. F. Wood, and R. D. Westbrook, Appl. Phys. Lett. 42, 258 (1983).

${ }^{3}$ J. M. Liu, H. Kurz, and N. Bloembergen, Appl.
Phys. Lett. 41, 693 (1982).

${ }^{4}$ C. V. Shank, R. Yen, and C. Hirliman, Phys. Rev. Lett. 50, 454 (1983).

${ }^{5}$ B. C. Larson, C. W. White, T. S. Noggle, and

D. Mills, Phys. Rev. Lett. 48, 387 (1982).

${ }^{6}$ B. C. Larson, C. W. White, T. S. Noggle, J. F. Barhorst, and D. M. Mills, Appl. Phys. Lett. $\underline{42}, 232$ (1983).

${ }^{7}$ C. V. Shank, R. Yen, and C. Hirliman, Phys. Rev. Lett. 51,900 (1983).

${ }^{8} \mathrm{H} . \overline{\mathrm{W}}$. Lo and A. Campaan, Phys. Rev. Lett. $\underline{44}$, 1604 (1980).

${ }^{9}$ D. von der Linde and G. Wartmann, Appl. Phys. Lett. 41, 700 (1982).

${ }^{10} \mathrm{D}$. von der Linde, G. Wartmann, and A. Compaan, Appl. Phys. Lett. $\underline{43}, 613$ (1983).

${ }^{11}$ A. Pospiesczyk, M. A. Harith, and B. Stritzker, J. Appl. Phys. 54, 3176 (1983).

${ }^{12} \mathrm{~T}$. Hosokowa, H. Fujioka, and K. Ura, Rev. Sci. Instrum. $\underline{49}, 1293$ (1978).

${ }^{13} \mathrm{C}$. Mourou and S. Williamson, Appl. Phys. Lett. 41, 44 (1982).

${ }^{14} \mathrm{D}$. Haneman, in Surface Physics of Phosphors and Semiconductors, edited by C. G. Scott and C. E. Reed (Academic, London, 1975), pp. 58-65.

${ }^{15}$ Literature results indicate that each laser shot does incremental optical damage to $\mathrm{Ge}$; moreover Ge shows a marked tendency to form permanent surface ripples, especially at the pump laser fluences used in this work [D. J. Ehrlich, S. R. J. Brueck, and J. Y. Tsao, Appl. Phys. Lett. 41, 630 (1982)]. Our observations show, however, that these processes have negligible effect on the LEED intensity until the damage is extreme.

${ }^{16}$ International Tables for X-Ray Crystallography, edited by G. Fulley (Kynoch, Birmingham, England, 1972), Vol. 3, p. 241.

${ }^{17}$ The intensity data at $140 \mathrm{eV}$ can be used to extract a Debye temperature for the surface vibrational states. This is found to be $195^{\circ} \mathrm{K}$, in good agreement with the Debye temperature of $290^{\circ} \mathrm{K}$ (see Ref. 14) when the latter is divided by $\sqrt{2}$ to account for the lowered surface vibrational frequencies. 